\title{
Applications of differential equations to characterize the base of warped product submanifolds of cosymplectic space forms
}

\author{
Akram Ali $^{1}$, Fatemah Mofarreh ${ }^{2 *}$ (D) Wan Ainun Mior Othman ${ }^{3}$ and Dhriti Sundar Patra ${ }^{4}$
}

"Correspondence:

fyalmofarrah@pnu.edu.sa

${ }^{2}$ Mathematical Science

Department, Faculty of Science,

Princess Nourah bint Abdulrahman

University, 11546 Riyadh, Saudi

Arabia

Full list of author information is

available at the end of the article

\begin{abstract}
In the present, we first obtain Chen-Ricci inequality for C-totally real warped product submanifolds in cosymplectic space forms. Then, we focus on characterizing spheres and Euclidean spaces, by using the Bochner formula and a second-order ordinary differential equation with geometric inequalities. We derive the characterization for the base of the warped product via the first eigenvalue of the warping function. Also, it proves that there is an isometry between the base $\mathbb{N}_{1}$ and the Euclidean sphere $\mathbb{S}^{m_{1}}$ under some different extrinsic conditions.
\end{abstract}

Keywords: Warped product submanifolds; Cosymplectic space forms; Obata differential equation; Isometric; Geometric inequalities

\section{Introduction and motivations}

The seminal work of Obata [30] has become a basic tool of investigation in geometric analysis. Obata [30] provided a characterization theorem for the standard sphere in terms of a differential equation, nowadays famous as Obata equation. If $\left(\Omega^{n}, g\right)$ is a complete manifold, then the function $\omega$ is nonconstant and satisfying the ODE

$$
\operatorname{Hess}(\omega)+c \omega g=0
$$

if and only if there is an isometry between $\left(\Omega^{n}, g\right)$ and the sphere $\mathbb{S}^{n}(c)$, where $c$ denotes the sectional curvature. If $c=1$, then $\left(\Omega^{n}, g\right)$ and the unit sphere $\mathbb{S}^{n}$ are congruent. Many investigations have been dedicated to this subject, and therefore, characterization of the Euclidean space $\mathbb{R}^{n}$, the Euclidean sphere $\mathbb{S}^{n}$, and the complex projective space $\mathbb{C} P^{n}$ are recognized fields in differential geometry and studied in some researches, e.g., [1-10, 1315, 20-25]. In particular, the Euclidean space $\mathbb{R}^{n}$ is designated through the differential equation $\nabla^{2} \omega=c g$, where $c$ is a positive constant, which was proven by Tashiro [32]. In [27], Lichnerowicz established that if the first nonzero eigenvalue $\mu_{1}$ of Laplace operator of the compact manifold $\left(\Omega^{n}, g\right)$ with Ric $\geq n-1$ is $\mu_{1}=n$, then $\left(\Omega^{n}, g\right)$ is isometric to the sphere $\mathbb{S}^{n}$. Hence, Obata's theorem can be utilized to address the equality condition of Lichnerowicz's eigenvalue. Deshmukh-Al-Solamy [25] proved that an $n$-dimensional

(c) The Author(s) 2020. This article is licensed under a Creative Commons Attribution 4.0 International License, which permits use sharing, adaptation, distribution and reproduction in any medium or format, as long as you give appropriate credit to the original author(s) and the source, provide a link to the Creative Commons licence, and indicate if changes were made. The images or other third party material in this article are included in the article's Creative Commons licence, unless indicated otherwise in a credit line to the material. If material is not included in the article's Creative Commons licence and your intended use is not permitted by statutory regulation or exceeds the permitted use, you will need to obtain permission directly from the copyright holder. To view a copy of this licence, visit http://creativecommons.org/licenses/by/4.0/. 
Riemannian manifold $\left(\Omega^{n}, g\right)$ satisfying $0<$ Ric $\leq(n-1)\left(2-\frac{n c}{\mu_{1}} c\right)$ for a constant $c$, where $\mu_{1}$ is the first eigenvalue of the Laplacian, is isometric to $\mathbb{S}^{n}(c)$ if $\Omega^{n}$ admits a nonzero conformal gradient vector field. They also proved that if $\Omega^{n}$ is an Einstein manifold such that Einstein constant is $\mu=(n-1) c$, then $\Omega^{n}$ is isometric to $\mathbb{S}^{n}(c)$ with $c>0$ if it admits a conformal gradient vector field. Taking account of ODE (1.1), Barros et al. [11] showed that the gradient almost Ricci soliton $\left(\Omega^{n}, g, \nabla \omega, \lambda\right)$ that is compact is isometric to the Euclidean sphere with Codazzi-Ricci tensor and constant sectional curvature. For more terminology of Obata equation, see [30]. Motivated by the previous studies, we will establish the following results:

Theorem 1.1 Let $\Upsilon: \Omega^{n}=\mathbb{N}_{1} \times_{f} \mathbb{N}_{2} \rightarrow \mathbb{M}^{2 m+1}(\epsilon)$ be a C-totally real isometric embedding of the warped product submanifold $\Omega^{n}$ into a cosymplectic space form $\mathbb{M}^{2 m+1}(\epsilon)$ with nonnegative Ricci curvature. Then, the compact and minimal base $\mathbb{N}_{1}$ is isometric to the Euclidean sphere $\mathbb{S}^{m_{1}}\left(\sqrt{\frac{\lambda_{1}}{m_{1}}}\right)$ if the following equality holds:

$$
|\operatorname{Hess}(\omega)|^{2}=\frac{\lambda_{1}}{m_{1} m_{2}}\left\{\epsilon\left(1-n-m_{1} m_{2}\right)-n^{2}|\mathbb{H}|^{2}\right\},
$$

where $\lambda_{1}>0$ is the eigenvalue connected to the eigenfunction $\omega=\ln f$ and $\operatorname{Hess}(\omega)$ is a Hessian tensor for the function $\omega$. Moreover, here the constant curvature $c$ is equal to $\sqrt{\frac{\lambda_{1}}{m_{1}}}$. In particular, if $\lambda_{1}=m_{1}$ satisfies the condition

$$
|\operatorname{Hess}(\omega)|^{2}=\frac{1}{m_{2}}\left\{\epsilon\left(1-n-\lambda_{1} m_{2}\right)-n^{2}|\mathbb{H}|^{2}\right\}
$$

then the base $\mathbb{N}_{1}$ is isometric to the standard sphere $\mathbb{S}^{m_{1}}$.

From the Bochner formula, we are able to prove the following result:

Theorem 1.2 Let $\Upsilon: \Omega^{n}=\mathbb{N}_{1} \times_{f} \mathbb{N}_{2} \longrightarrow \mathbb{M}^{2 m+1}(\epsilon)$ be a C-totally real isometric embedding for the warped product submanifold $\Omega^{n}$ to the cosymplectic space form $\mathbb{M}^{2 m+1}(\epsilon)$ with base $\mathbb{N}_{1}$ being minimal and compact. If the Ricci curvature of $\Omega^{n}$ is nonnegative, then $\mathbb{N}_{1}$ is isometric to the sphere $\mathbb{S}^{m_{1}}(c)$ with constant curvature equal to $c=\sqrt{\frac{\lambda_{1}}{m_{1}}}$ if the following equality holds:

$$
|\mathbb{H}|^{2}=\frac{\epsilon\left(1-m_{1} m_{2}-n\right)}{n^{2}},
$$

where $\lambda_{1}>0$ is an eigenvalue associated with the eigenfunction $\omega=\ln f$. Moreover, $n=$ $\operatorname{dim} \Omega, m_{1}=\operatorname{dim} \mathbb{N}_{1}$, and $m_{2}=\operatorname{dim} \mathbb{N}_{2}$.

Remark 1.1 For examples of $C$-totally real isometric immersions from warped product manifolds, see [31, 34].

\section{Preliminaries and notations}

Let $(\widetilde{\mathbb{M}}, g)$ be an odd-dimensional $C^{\infty}$-manifold equipped with an almost contact structure $(\varphi, \kappa, \eta)$ such that

$$
\varphi^{2}=-I+\eta \otimes \kappa, \quad \eta(\kappa)=1,
$$




$$
\begin{aligned}
& \varphi(\kappa)=0, \quad \eta \circ \varphi=0, \\
& g\left(\varphi \mathbb{W}_{1}, \varphi \mathbb{W}_{2}\right)=g\left(\mathbb{W}_{1}, \mathbb{W}_{2}\right)-\eta\left(\mathbb{W}_{1}\right) \eta\left(\mathbb{W}_{2}\right), \\
& \eta\left(\mathbb{W}_{1}\right)=g\left(\mathbb{W}_{1}, \kappa\right)
\end{aligned}
$$

for any $\mathbb{W}_{1}, \mathbb{W}_{2} \in \Gamma(T \tilde{\mathbb{M}})$. Of course, the notations are well known: $\kappa$ is a structure vector field, $(1,1)$-type tensor field is denoted by $\varphi$, and $\eta$ is the dual 1 -form. Moreover, the tonsorial equation for a cosymplectic manifold [7] with the structure $(\varphi, \kappa, \eta)$ is given by

$$
\left(\widetilde{\nabla}_{\mathbb{W}_{1}} \varphi\right) \mathbb{W}_{2}=0
$$

if we choose two vector fields $\mathbb{W}_{1}, \mathbb{W}_{2}$ over $\widetilde{\mathbb{M}}$ such that $\widetilde{\nabla}$ is the Riemannian connection corresponding to $g$. Assume that $\widetilde{\mathbb{M}}^{2 m+1}(\epsilon)$ is a cosymplectic space form with constant $\varphi$-sectional curvature $\epsilon$, then its curvature tensor $\widetilde{R}$ is

$$
\begin{aligned}
\widetilde{R}\left(\mathbb{W}_{1}, \mathbb{W}_{2}, \mathbb{W}_{3}, \mathbb{W}_{4}\right)= & \frac{\epsilon}{4}\left\{g\left(\mathbb{W}_{2}, \mathbb{W}_{3}\right) g\left(\mathbb{W}_{1}, \mathbb{W}_{4}\right)-g\left(\mathbb{W}_{1}, \mathbb{W}_{3}\right) g\left(\mathbb{W}_{2}, \mathbb{W}_{4}\right)\right. \\
& +\eta\left(\mathbb{W}_{1}\right) \eta\left(\mathbb{W}_{3}\right) g\left(\mathbb{W}_{2}, \mathbb{W}_{4}\right)+\eta\left(\mathbb{W}_{4}\right) \eta\left(\mathbb{W}_{2}\right) g\left(\mathbb{W}_{1}, \mathbb{W}_{3}\right) \\
& -\eta\left(\mathbb{W}_{2}\right) \eta\left(\mathbb{W}_{3}\right) g\left(\mathbb{W}_{1}, \mathbb{W}_{4}\right)-\eta\left(\mathbb{W}_{1}\right) g\left(\mathbb{W}_{2}, \mathbb{W}_{3}\right) \eta\left(\mathbb{W}_{4}\right) \\
& +g\left(\varphi \mathbb{W}_{2}, \mathbb{W}_{3}\right) g\left(\varphi \mathbb{W}_{1}, \mathbb{W}_{4}\right)-g\left(\varphi \mathbb{W}_{1}, \mathbb{W}_{3}\right) g\left(\varphi \mathbb{W}_{2}, \mathbb{W}_{4}\right) \\
& \left.+2 g\left(\mathbb{W}_{1}, \varphi \mathbb{W}_{2}\right) g\left(\varphi \mathbb{W}_{3}, \mathbb{W}_{4}\right)\right\}
\end{aligned}
$$

for all $\mathbb{W}_{1}, \mathbb{W}_{2}, \mathbb{W}_{3}, \mathbb{W}_{4} \in \Gamma(T \tilde{\mathbb{M}})$. Moreover, if the structure vector field $\kappa$ belongs to the normal space of $\Omega^{n}$, then $\Omega^{n}$ is said to be a $C$-totally real submanifold; for more details, see $[7,26,28,32,33]$. It should be noted that the curvature tensor $\widetilde{R}$ for $\Omega^{n}$ in cosymplectic space form $\widetilde{\mathbb{M}}^{2 m+1}(\epsilon)$ is defined as

$$
\widetilde{R}\left(\mathbb{W}_{1}, \mathbb{W}_{2}, \mathbb{W}_{3}, \mathbb{W}_{4}\right)=\frac{\epsilon}{4}\left\{g\left(\mathbb{W}_{2}, \mathbb{W}_{3}\right) g\left(\mathbb{W}_{1}, \mathbb{W}_{4}\right)-g\left(\mathbb{W}_{1}, \mathbb{W}_{3}\right) g\left(\mathbb{W}_{2}, \mathbb{W}_{4}\right)\right\}
$$

Suppose $\Omega^{n}$ is a Riemannian submanifold of a Riemannian manifold $\widetilde{\mathbb{M}}^{2 m+1}$ considering induced metric $g, \nabla$ and $\nabla^{\perp}$ are connections along $T \Omega$ and $T^{\perp} \Omega$ of $\Omega^{n}$, where $T \Omega$ is a tangent bundle and $T^{\perp} \Omega$ is the normal bundle of $\Omega^{n}$. Therefore, the Gauss and Weingarten formulae are written as $\widetilde{\nabla}_{\mathbb{W}_{1}} \mathbb{W}_{2}=\nabla_{\mathbb{W}_{1}} \mathbb{W}_{2}+\zeta\left(\mathbb{W}_{1}, \mathbb{W}_{2}\right)$ and $\widetilde{\nabla}_{\mathbb{W}_{1}} \xi=-A_{\xi} \mathbb{W}_{1}+\nabla_{\mathbb{W}_{1}}^{\perp} \xi$, respectively, for $\mathbb{W}_{1}, \mathbb{W}_{2} \in \mathfrak{X}(T \Omega)$ and $\xi \in \mathfrak{X}\left(T^{\perp} \Omega\right)$. Note that $\zeta$ and $A_{\xi}$ denote the second fundamental form and shape operator, respectively, for the embedding of $\Omega^{n}$ to $\mathbb{M}^{2 m+1}$, and they are governed by the relation $g\left(\zeta\left(\mathbb{W}_{1}, \mathbb{W}_{2}\right), \xi\right)=g\left(A_{\xi} \mathbb{W}_{1}, \mathbb{W}_{2}\right)$. The Gauss equation is

$$
\begin{aligned}
R\left(\mathbb{W}_{1}, \mathbb{W}_{2}, \mathbb{W}_{3}, \mathbb{W}_{4}\right)= & \widetilde{R}\left(\mathbb{W}_{1}, \mathbb{W}_{2}, \mathbb{W}_{3}, \mathbb{W}_{4}\right)+g\left(\zeta\left(\mathbb{W}_{1}, \mathbb{W}_{4}\right), \zeta\left(\mathbb{W}_{2}, \mathbb{W}_{3}\right)\right) \\
& -g\left(\zeta\left(\mathbb{W}_{1}, \mathbb{W}_{3}\right), \zeta\left(\mathbb{W}_{2}, \mathbb{W}_{4}\right)\right)
\end{aligned}
$$

for any $\mathbb{W}_{1}, \mathbb{W}_{2}, \mathbb{W}_{3}, \mathbb{W}_{4} \in \mathfrak{X}(\tilde{\mathbb{M}})$, where the curvature tensors of $\tilde{\mathbb{M}}^{2 m+1}$ and $\Omega^{n}$ are represented by $\widetilde{R}$ and $R$. Also, the mean curvature $\mathbb{H}$ of $\Omega^{n}$ is calculated as $\mathbb{H}=\frac{1}{n} \operatorname{trace}(\zeta)$, and $\Omega^{n}$ is totally umbilical if $\zeta\left(\mathbb{W}_{1}, \mathbb{W}_{2}\right)=g\left(\mathbb{W}_{1}, \mathbb{W}_{2}\right) \mathbb{H}$ and totally geodesic if $\zeta\left(\mathbb{W}_{1}, \mathbb{W}_{2}\right)=0$, 
for any $\mathbb{W}_{1}, \mathbb{W}_{2} \in \mathfrak{X}(\Omega)$. Furthermore, $\Omega^{n}$ is minimal if $\mathbb{H}=0$. Here,

$$
\mathscr{N}_{x}=\left\{X \in T_{x} \Omega \mid \zeta\left(\mathbb{W}_{1}, \mathbb{W}_{2}\right)=0 \text { for all } \mathbb{W}_{2} \in T_{x} \Omega\right\}
$$

gives the second fundamental form kernel of $\Omega^{n}$ over $x$. If the plane section is spanned by $e_{\ell}$ and $e_{\gamma}$ over $x$ in $\widetilde{\mathbb{M}}^{2 m+1}$ then such a curvature is called sectional curvature and it is denoted by $\widetilde{\mathbb{K}}_{\ell \gamma}=\widetilde{\mathbb{K}}\left(e_{\ell} \wedge e_{\gamma}\right)$. The relation between the scalar curvature $\widetilde{\tau}\left(T_{x} \widetilde{\mathbb{M}}\right)$ of $\widetilde{\mathbb{M}}^{2 m+1}$ and $\widetilde{\mathbb{K}}\left(e_{\ell} \wedge e_{\gamma}\right)$ at some $x$ in $\widetilde{\mathbb{M}}^{2 m+1}$ is represented by

$$
\tilde{\tau}\left(T_{x} \tilde{\mathbb{M}}\right)=\sum_{1 \leq \ell<\gamma \leq 2 m+1} \widetilde{\mathbb{K}}_{\ell \gamma}
$$

The equality in (2.8) is equivalent to the following:

$$
2 \widetilde{\tau}\left(T_{x} \tilde{\mathbb{M}}\right)=\sum_{1 \leq \ell<\gamma \leq n} \widetilde{\mathbb{K}}_{\ell \gamma}, \quad 1 \leq \ell, \gamma \leq n .
$$

The latter relation will be utilized in the subsequent proofs. Similarly, the scalar curvature $\widetilde{\tau}\left(L_{x}\right)$ of an $L$-plane is expressed as

$$
\tilde{\tau}\left(L_{x}\right)=\sum_{1 \leq \ell<\gamma \leq m} \widetilde{\mathbb{K}}_{\ell \gamma}
$$

Let $\left\{e_{1}, \ldots, e_{n}\right\}$ be an orthonormal frame of the tangent space $T_{x} \Omega$ and $e_{r}=\left(e_{n+1}, \ldots, e_{2 m+1}\right)$ be an orthonormal frame of the normal space $T^{\perp} \Omega$. Hence we have

$$
\begin{aligned}
& \zeta_{\ell \gamma}^{r}=g\left(\zeta\left(e_{\ell}, e_{\gamma}\right), e_{r}\right) \quad \text { and } \\
& \|\zeta\|^{2}=\sum_{\ell, \gamma=1}^{n} g\left(\zeta\left(e_{\ell}, e_{\gamma}\right), \zeta\left(e_{\ell}, e_{\gamma}\right)\right)=\sum_{\ell, \gamma=1}^{n}\left(\zeta_{\ell \gamma}^{r}\right)^{2} .
\end{aligned}
$$

Let $\mathbb{K}_{\ell \gamma}$ and $\widetilde{\mathbb{K}}_{\ell \gamma}$ be the sectional curvature of a submanifold $\Omega^{n}$ and $\widetilde{\mathbb{M}}^{2 m+1}$, respectively, then we have following relation due to the Gauss equation (2.6):

$$
\begin{aligned}
2 \tau\left(T_{x} \Omega^{n}\right) & =\mathbb{K}_{\ell \gamma}=2 \widetilde{\tau}\left(T_{x} \Omega^{n}\right)+\sum_{r=n+1}^{2 m+1}\left(\zeta_{\ell \ell}^{r} \zeta_{\gamma \gamma}^{r}-\left(\zeta_{\ell \gamma}^{r}\right)^{2}\right) \\
& =\widetilde{\mathbb{K}}_{\ell \gamma}+\sum_{r=n+1}^{2 m+1}\left(\zeta_{\ell \ell}^{r} \zeta_{\gamma \gamma}^{r}-\left(\zeta_{\ell \gamma}^{r}\right)^{2}\right) .
\end{aligned}
$$

Furthermore, the Ricci tensor is defined for an orthonormal basis $\left\{e_{1}, \ldots, e_{n}\right\}$ of $\Omega^{n}$ as

$$
\widetilde{S}\left(\mathbb{W}_{1}, \mathbb{W}_{2}\right)=\sum_{i=1}^{n}\left\{\widetilde{g}\left(\widetilde{R}\left(e_{\ell}, \mathbb{W}_{1}\right) \mathbb{W}_{2}, e_{\ell}\right)\right\}, \quad \mathbb{W}_{1}, \mathbb{W}_{2} \in \Gamma\left(T_{x} \Omega^{n}\right)
$$

Using the distinct indices for vector fields $\left\{e_{1}, \ldots, e_{n}\right\}$ on $\Omega^{n}$ from $e_{u}$, which is governed by $W$, then the Ricci curvature is given as

$$
\mathscr{R} i c(\mathbb{W})=\sum_{\substack{\ell=1 \\ \ell \neq u}}^{n} \mathbb{K}\left(e_{\ell} \wedge e_{u}\right) .
$$


Therefore, equation (2.9) can be written as

$$
\tilde{\tau}\left(T_{x} \Omega^{n}\right)=\sum_{1 \leq \ell<\gamma \leq n} \mathbb{K}\left(e_{\ell} \wedge e_{\gamma}\right)=\frac{1}{2} \sum_{A=1}^{n} \mathscr{R} i c\left(e_{u}\right) .
$$

Hence,

$$
2 \tilde{\tau}\left(T_{x} \Omega^{n}\right)=\sum_{1 \leq \ell<\gamma \leq n} \mathbb{K}\left(e_{\ell} \wedge e_{\gamma}\right)=\frac{1}{2} \sum_{u=1}^{n} \mathscr{R} i c\left(e_{u}\right)
$$

which will be frequently used in the following study. For a $k$-plane $L_{k}$ of $T_{x} \Omega^{n}$, suppose $\left\{e_{1}, \ldots, e_{k}\right\}$ is an orthonormal frame of $L_{k}$, thus for a fixed $u \in\{1, \ldots, k\}$, the $k$-Ricci curvature $\widetilde{R} i c_{L_{k}}\left(e_{u}\right)$ of $L_{k}$ is defined by

$$
\widetilde{R}_{L_{k}}\left(e_{u}\right)=\sum_{\substack{\ell=1 \\ \ell \neq u}}^{k} \mathbb{K}\left(e_{\ell} \wedge e_{u}\right)
$$

The gradient squared-norm of the positive smooth function $\omega$ of the orthonormal basis $\left\{e_{1}, \ldots, e_{n}\right\}$ is given by

$$
\|\nabla \omega\|^{2}=\sum_{i=1}^{n}\left(e_{i}(\omega)\right)^{2}
$$

Assume that $\mathbb{N}_{1}$ and $\mathbb{N}_{2}$ are Riemannian manifolds with Riemannian metrics $g_{1}$ and $g_{2}$, respectively. Suppose $f$ is a differentiable function in $\mathbb{N}_{1}$. Then, the manifold $\mathbb{N}_{1} \times \mathbb{N}_{2}$ equipped with the Riemannian metric $g=g_{1}+f^{2} g_{2}$ is referred to as the warped product manifold and defined as $\Omega^{n}=\mathbb{N}_{1} \times_{f} \mathbb{N}_{2}$ (for details, see [17]). Assume that $\Omega^{n}=\mathbb{N}_{1} \times_{f} \mathbb{N}_{2}$ is a nontrivial warped product, then $\forall \mathbb{W}_{1} \in \Gamma\left(\mathbb{N}_{1}\right)$ and $\mathbb{W}_{2} \in \Gamma\left(\mathbb{N}_{2}\right)$, we have

$$
\nabla_{\mathbb{W}_{2}} \mathbb{W}_{1}=\nabla_{\mathbb{W}_{1}} \mathbb{W}_{2}=\left(\mathbb{W}_{1} \ln f\right) \mathbb{W}_{2}
$$

The following relation was proved in (see (3.3) in [17]) as follows:

$$
\sum_{\ell=1}^{m_{1}} \sum_{\gamma=1}^{m_{2}} K\left(e_{\ell} \wedge e_{\gamma}\right)=\frac{m_{2} \Delta(f)}{f}=m_{2}\left(\Delta(\ln f)-\|\nabla(\ln f)\|^{2}\right)
$$

Remark 2.1 $\Omega^{n}=\mathbb{N}_{1} \times_{f} \mathbb{N}_{2}$ is a Riemannian product manifold if $f$ is a constant.

Lemma 2.1 Suppose $\Upsilon: \Omega^{n}=\mathbb{N}_{1} \times_{f} \mathbb{N}_{2} \longrightarrow \mathbb{M}^{2 m+1}(\epsilon)$ is a C-totally real warped product immersed submanifold into a cosymplectic space form $\widetilde{\mathbb{M}}^{2 m+1}$ whose base $\mathbb{N}_{1}$ is minimal. Then, for all unit vectors $\mathbb{W} \in T_{x} \Omega^{n}$, the Ricci inequality

$$
\mathscr{R} i c(\mathbb{W})+m_{2} \Delta \ln f \leq \frac{n^{2}}{4}\|\mathbb{H}\|^{2}+m_{2}\|\nabla \ln f\|^{2}+\frac{\epsilon}{4}\left\{m_{1} m_{2}+n-1\right\}
$$

holds, where $m_{1}=\operatorname{dim} \mathbb{N}_{1}$ and $m_{2}=\operatorname{dim} \mathbb{N}_{2}$. Furthermore, 
(1) In case that $H(x)=0$, for $x \in \Omega^{n}$ there exists a unit vector $\mathbb{W}$ satisfying the equality in (2.21) if and only if $\Omega^{n}$ is mixed totally geodesic and $\mathbb{W}$ lies in $\mathscr{N}_{x}$ at $x$.

If $\Omega^{n}$ is $\mathbb{N}_{1}$-minimal, then

(a) The equality in (2.21) remains true for any unit tangent vectors at $\mathbb{N}_{1}$ and any $x \in \Omega^{n} \Longleftrightarrow \Omega^{n}$ is totally geodesic and $\mathbb{N}_{1}$-totally geodesic WP in $\tilde{\mathbb{M}}^{2 m+1}$.

(b) The equality in (2.21) remains true for any unit tangent vectors at $\mathbb{N}_{2}$ and any $x \in \Omega^{n} \Longleftrightarrow \Omega^{n}$ is totally geodesic, and either an $\mathbb{N}_{2}$-totally geodesic WPS, or an $\mathbb{N}_{2}$-totally umbilical WPS in $x \in \widetilde{\mathbb{M}}^{2 m+1}$ such that $\operatorname{dim} \mathbb{N}_{2}=2$.

(2) The equality in (2.21) is satisfied for any unit tangent vectors at $\Omega^{n}$ and any $x \in \Omega^{n} \Longleftrightarrow \Omega^{n}$ is either totally geodesic or totally umbilical, mixed totally geodesic and $\mathbb{N}_{1}$-totally geodesic WPS such that $\operatorname{dim} \mathbb{N}_{2}=2$.

Proof Assume that $\Omega^{n}$ is $\mathbb{N}_{1}$-minimal $C$-totally real warped product. An analogous technique will be used for similar cases. Utilizing Gauss equation (2.6), we derive

$$
n^{2}\|\mathbb{H}\|^{2}=2 \tau\left(T_{x} \Omega^{n}\right)+\|\zeta\|^{2}-2 \widetilde{\tau}\left(T_{x} \Omega^{n}\right) .
$$

Assume $\left\{e_{1}, \ldots, e_{m_{1}}, e_{m_{1}+1}, \ldots, e_{n}\right\}$ are the local orthonormal frame fields of $\mathfrak{X}\left(\tilde{\mathbb{M}}^{2 m+1}\right)$ in which $\left\{e_{1}, \ldots, e_{m_{1}}\right\}$ are tangent to $\mathbb{N}_{1}$ and $\left\{e_{m_{1}+1}, \ldots, e_{n}\right\}$ are tangent to $\mathbb{N}_{2}$. Hence, for the unit tangent vector $\mathbb{W}=e_{u} \in\left\{e_{1}, \ldots, e_{n}\right\}$, we can expand (2.22) as follows:

$$
\begin{aligned}
n^{2}\|\mathbb{H}\|^{2}= & 2 \tau\left(T_{x} \Omega^{n}\right)+\frac{1}{2} \sum_{r=n+1}^{2 m+1}\left\{\left(\zeta_{11}^{r}+\cdots+\zeta_{n n}^{r}-\zeta_{u u}^{r}\right)^{2}+\left(\zeta_{u u}^{r}\right)^{2}\right\} \\
& -\sum_{r=n+1}^{2 m+1} \sum_{1 \leq \ell \neq \gamma \leq n} \zeta_{\ell \ell}^{r} \zeta_{\gamma \gamma}^{r}-2 \tilde{\tau}\left(T_{x} \Omega^{n}\right) .
\end{aligned}
$$

It is equivalent to

$$
\begin{aligned}
n^{2}\|\mathbb{H}\|^{2}= & 2 \tau\left(T_{x} \Omega^{n}\right)+\sum_{r=n+1}^{2 m+1}\left\{\left(\zeta_{11}^{r}+\cdots+\zeta_{n n}^{r}\right)^{2}+\left(2 \zeta_{u u}^{r}-\left(\zeta_{11}^{r}+\cdots+\zeta_{n n}^{r}\right)\right)^{2}\right\} \\
& +2 \sum_{r=n+1}^{2 m+1} \sum_{1 \leq \ell<\gamma \leq n}\left(\zeta_{\ell \gamma}^{r}\right)^{2}-2 \sum_{r=n+1}^{2 m+1} \sum_{1 \leq \ell<\gamma \leq n} \zeta_{\ell}^{r} \zeta_{\gamma}^{r}-\frac{\epsilon}{4} n(n-1) .
\end{aligned}
$$

As we assumed the warped product submanifold $\Omega^{n}$ to be $\mathbb{N}_{1}$-minimal, we derive

$$
\begin{aligned}
n^{2}\|\mathbb{H}\|^{2}+\frac{\epsilon}{4} n(n-1) & \sum_{r=n+1}^{2 m+1}\left\{\left(\zeta_{m_{1}+1 m_{1}+1}^{r}+\cdots+\zeta_{n n}^{r}\right)^{2}+\left(2 \zeta_{u u}^{r}-\left(\zeta_{m_{1}+1 m_{1}+1}^{r}+\cdots+\zeta_{n n}^{r}\right)\right)^{2}\right\} \\
& +2 \tau\left(T_{x} \Omega^{n}\right)+\sum_{r=n+1}^{2 m+1} \sum_{1 \leq \ell<\gamma \leq n}\left(\zeta_{\ell \gamma}^{r}\right)^{2}-\sum_{r=n+1}^{2 m+1} \sum_{\substack{1 \leq \ell<\gamma \leq n \\
2 m}} \zeta_{\ell \ell}^{r} \zeta_{\gamma \gamma}^{r} \\
& +\sum_{r=n+1}^{2 m+1} \sum_{\substack{a=1 \\
a \neq u}}\left(\zeta_{a u}^{r}\right)^{2}+\sum_{r=n+1}^{2 m+1} \sum_{\substack{1 \leq \ell<\gamma \leq n \\
\ell, \gamma \neq u}}\left(\zeta_{\ell \gamma}^{r}\right)^{2}-\sum_{\substack { r=n+1 \\
\begin{subarray}{c}{1 \leq \ell<\gamma \leq n \\
\ell, \gamma \neq u{ r = n + 1 \\
\begin{subarray} { c } { 1 \leq \ell < \gamma \leq n \\
\ell , \gamma \neq u } }\end{subarray}} \zeta_{\ell \ell}^{r} \zeta_{\gamma \gamma}^{r} .
\end{aligned}
$$


In view of (2.12), we obtain

$$
\sum_{r=n+1}^{2 m+1} \sum_{\substack{1 \leq \ell<\gamma \leq n \\ \ell, \gamma \neq u}}\left(\zeta_{\ell \gamma}^{r}\right)^{2}-\sum_{r=n+1}^{2 m+1} \sum_{\substack{1 \leq \ell<\gamma \leq n \\ \ell, \gamma \neq u}} \zeta_{\ell \ell}^{r} \zeta_{\gamma \gamma}^{r}=\sum_{\substack{1 \leq \ell<\gamma \leq n \\ \ell, \gamma \neq A}} \widetilde{\mathbb{K}}_{\ell \gamma}-\sum_{\substack{1 \leq \ell<\gamma \leq n \\ \ell, \gamma \neq A}} \mathbb{K}_{\ell \gamma} .
$$

From fact that the base $\mathbb{N}_{1}$ is minimal, and putting (2.24) in (2.23), we deduce

$$
\begin{aligned}
& \frac{1}{2} n^{2}\|\mathbb{H}\|^{2}+\frac{\epsilon}{4} n(n-1)=2 \tau\left(T_{x} \Omega^{n}\right)+\frac{1}{2} \sum_{r=n+1}^{2 m+1}\left(2 \zeta_{u u}^{r}-\left(\zeta_{m_{1}+1 m_{1}+1}^{r}+\cdots+\zeta_{n n}^{r}\right)\right)^{2} \\
& +\sum_{r=n+1}^{2 m+1} \sum_{1 \leq \ell<\gamma \leq n}\left(\zeta_{\ell \gamma}^{r}\right)^{2}-\sum_{r=n+1}^{m} \sum_{\substack{1 \leq \ell<\gamma \leq n \\
\ell, \gamma \neq u}} \zeta_{\ell \ell}^{r} \zeta_{\gamma \gamma}^{r} \\
& +\sum_{r=n+1}^{2 m+1} \sum_{\substack{a=1, a \neq u}}\left(\zeta_{a u}^{r}\right)^{2}+\sum_{\substack{1 \leq \ell<\gamma \leq n \\
\ell, \gamma \neq u}} \widetilde{\mathbb{K}}_{\ell \gamma}-\sum_{\substack{1 \leq \ell<\gamma \leq n \\
\ell, \gamma \neq u}} \mathbb{K}_{\ell \gamma}
\end{aligned}
$$

On the other hand, using (2.8), we define

$$
\begin{aligned}
\tau\left(T_{x} \Omega^{n}\right)= & \sum_{1 \leq \ell<\gamma \leq n} \mathbb{K}\left(e_{\ell} \wedge e_{\gamma}\right) \\
= & \sum_{i=1}^{m_{1}} \sum_{j=m_{1}+1}^{n} \mathbb{K}\left(e_{i} \wedge e_{j}\right)+\sum_{1 \leq i<k \leq m_{1}} \mathbb{K}\left(e_{i} \wedge e_{k}\right) \\
& +\sum_{m_{1}+1 \leq l<o \leq n} \mathbb{K}\left(e_{l} \wedge e_{o}\right) .
\end{aligned}
$$

From (2.20) and (2.8), we get

$$
\tau\left(T_{x} \Omega^{n}\right)=\frac{m_{2} \Delta f}{f}+\tau\left(T_{x} \mathbb{N}_{1}^{m_{1}}\right)+\tau\left(T_{x} \mathbb{N}_{2}^{m_{2}}\right)
$$

Now it suffices to combine (2.25), (2.26), (2.27), and use (2.11) to arrive at the following result:

$$
\begin{aligned}
\frac{1}{2} n^{2}\|\mathbb{H}\|^{2}+\frac{\epsilon}{4} n(n-1)= & \frac{m_{2} \Delta f}{f}-2 \tilde{\tau}\left(T_{x} \Omega^{n}\right)+\sum_{\substack{1 \leq \ell<\gamma \leq n \\
\ell, \gamma \neq u}} \widetilde{\mathbb{K}}_{\ell \gamma}+\tilde{\tau}\left(T_{x} \mathbb{N}_{1}^{m_{1}}\right)+\tilde{\tau}\left(T_{x} \mathbb{N}_{2}^{m_{2}}\right) \\
& +\sum_{r=n+1}^{2 m+1}\left\{\sum_{1 \leq \ell<\gamma \leq n}\left(\zeta_{\ell \gamma}^{r}\right)^{2}-\sum_{\substack{1 \leq \ell<\gamma \leq n \\
\ell, \gamma \neq u}} \zeta_{\ell \ell}^{r} \zeta_{\gamma \gamma}^{r}\right\} \\
& +\sum_{r=n+1}^{2 m+1} \sum_{\substack{a=1, a \neq u}}\left(\zeta_{a u}^{r}\right)^{2}+\sum_{r=n+1}^{m} \sum_{\substack{1 \leq i \neq j \leq p \\
\text { i }}}\left(\zeta_{i i}^{r} \zeta_{j j}^{r}-\left(\zeta_{i j}^{r}\right)^{2}\right)
\end{aligned}
$$




$$
\begin{aligned}
& +\sum_{r=n+1}^{2 m+1} \sum_{m_{1}+1 \leq s \neq t \leq n}\left(\zeta_{s s}^{r} \zeta_{t t}^{r}-\left(\zeta_{s t}^{r}\right)^{2}\right) \\
& +\frac{1}{2} \sum_{r=n+1}^{2 m+1}\left(2 \zeta_{u u}^{r}-\left(\zeta_{m_{1}+1 m_{1}+1}^{r}+\cdots+\zeta_{n n}^{r}\right)\right)^{2}
\end{aligned}
$$

Now we note that $e_{u}$ is either tangent to base $\mathbb{N}_{1}$ or to fiber $\mathbb{N}_{2}$. We show this by considering two cases.

Case $I$. Let $e_{u}$ be tangent to $\mathbb{N}_{1}$. Fix the unit tangent vector from $\left\{e_{1}, \ldots, e_{m_{1}}\right\}$ to be $e_{u}$, and consider $\mathbb{W}=e_{u}=e_{1}$. Then, from (2.14) and (2.28), we get

$$
\begin{aligned}
\frac{1}{2} n^{2} \| & H \|^{2}+\frac{\epsilon}{4} n(n-1) \\
\geq & \mathscr{R} i c(\mathbb{W})+\frac{m_{2} \Delta f}{f}-2 \widetilde{\tau}\left(T_{x} \Omega^{n}\right)+\tilde{\tau}\left(T_{x} \mathbb{N}_{1}^{m_{1}}\right)+\widetilde{\tau}\left(T_{x} \mathbb{N}_{2}^{m_{2}}\right) \\
& +\sum_{2 \leq \ell<\gamma \leq n} \widetilde{K}_{\ell \gamma}+\frac{1}{2} \sum_{r=n+1}^{2 m+1}\left(2 \zeta_{11}^{r}-\left(\zeta_{m_{1}+1 m_{1}+1}^{r}+\cdots+\zeta_{n n}^{r}\right)\right)^{2} \\
& +\sum_{r=n+1}^{2 m+1} \sum_{1 \leq \ell<\gamma \leq n}\left(\zeta_{\ell \gamma}^{r}\right)^{2}-\sum_{r=n+1}^{2 m+1}\left\{\sum_{1 \leq i<j \leq m_{1}}\left(\zeta_{i j}^{r}\right)^{2}+\sum_{m_{1}+1 \leq s<t \leq n}\left(\zeta_{s t}^{r}\right)^{2}\right\} \\
& +\sum_{r=n+1}^{2 m+1}\left\{\sum_{1 \leq i<j \leq m_{1}} \zeta_{i i}^{r} \zeta_{j j}^{r}+\sum_{r=n+1}^{2 m+1} \sum_{m_{1}+1 \leq s \neq t \leq n} \zeta_{s s}^{r} \zeta_{t t}^{r}-\sum_{2 \leq \ell<\gamma \leq n} \zeta_{\ell \ell}^{r} h_{\gamma \gamma}^{r}\right\} .
\end{aligned}
$$

Substituting $\mathbb{W}_{1}=\mathbb{W}_{2}=e_{\ell}$ and $\mathbb{W}_{2}=\mathbb{W}_{2}=e_{\gamma}$ for $1 \leq \ell, \gamma \leq n$ in (2.6), and summing up, we obtain

$$
\sum_{\ell, \gamma=1}^{n} \widetilde{R}\left(e_{\ell}, e_{\gamma}, e_{\ell}, e_{\gamma}\right)=\frac{\epsilon}{4} n(n-1) .
$$

Therefore, using (2.30) in (2.29), we obtain

$$
\begin{aligned}
\mathscr{R} i c(\mathbb{W}) \leq & \frac{n^{2}}{2}\|H\|^{2}-\frac{m_{2} \Delta f}{f}+\frac{\epsilon}{4}\left(m_{1} m_{2}+n-1\right) \\
& -\frac{1}{2} \sum_{r=n+1}^{m}\left(2 \zeta_{11}^{r}-\left(\zeta_{m_{1}+1 m_{1}+1}^{r}+\cdots+\zeta_{n n}^{r}\right)\right)^{2} \\
& +\sum_{r=n+1}^{2 m+1}\left\{\sum_{1 \leq i<j \leq m_{1}}\left(\zeta_{i j}^{r}\right)^{2}+\sum_{m_{1}+1 \leq s<t \leq n}\left(\zeta_{s t}^{r}\right)^{2}\right\} \\
& -\sum_{r=n+1}^{2 m+1}\left\{\sum_{1 \leq i<j \leq m_{1}} \zeta_{i i}^{r} \zeta_{j j}^{r}+\sum_{r=n+1}^{2 m+1} \sum_{m_{1}+1 \leq s \neq t \leq n} \zeta_{s s}^{r} \zeta_{t t}^{r}\right\} \\
& +\sum_{r=n+1}^{2 m+1} \sum_{2 \leq \ell<\gamma \leq n} \zeta_{\ell \ell}^{r} \zeta_{\gamma \gamma}^{r}-\sum_{r=n+1}^{2 m+1} \sum_{1 \leq \ell<\gamma \leq n}\left(\zeta_{\ell \gamma}^{r}\right)^{2} .
\end{aligned}
$$


The calculation of the last two terms of (2.31) implies

$$
\begin{aligned}
& \sum_{r=n+1}^{2 m+1}\left\{\sum_{1 \leq i<j \leq m_{1}}\left(\zeta_{i j}^{r}\right)^{2}+\sum_{m_{1}+1 \leq s<t \leq n}\left(\zeta_{s t}^{r}\right)^{2}\right\}-\sum_{r=n+1}^{2 m+1} \sum_{1 \leq \ell<\gamma \leq n}\left(\zeta_{\ell \gamma}^{r}\right)^{2} \\
& =\sum_{r=n+1}^{2 m+1} \sum_{\ell=1}^{m_{1}} \sum_{\gamma=m_{1}+1}^{n}\left(\zeta_{\ell \gamma}^{r}\right)^{2} .
\end{aligned}
$$

In a similar way, we obtain

$$
\begin{aligned}
& \sum_{r=n+1}^{2 m+1}\left\{\sum_{1 \leq i<j \leq m_{1}} \zeta_{i i}^{r} \zeta_{j j}^{r}+\sum_{r=n+1}^{m} \sum_{m_{1}+1 \leq s \neq t \leq n} \zeta_{s s}^{r} \zeta_{t t}^{r}-\sum_{2 \leq \ell<\gamma \leq n} \zeta_{\ell}^{r} \zeta_{\gamma}^{r}\right\} \\
& =\sum_{r=n+1}^{2 m+1}\left(\sum_{j=2}^{m_{1}} \zeta_{11}^{r} \zeta_{j j}^{r}-\sum_{\ell=2}^{m_{1}} \sum_{\gamma=m_{1}+1}^{n} \zeta_{\ell \ell}^{r} \zeta_{\gamma \gamma}^{r}\right) .
\end{aligned}
$$

Using (2.33) in (2.29) leads to

$$
\begin{aligned}
\mathscr{R} i c(\mathbb{W}) \leq & \frac{1}{2} n^{2}\|\mathbb{H}\|^{2}-\frac{m_{2} \Delta f}{f}+\frac{\epsilon}{4}\left(m_{1} m_{2}+n-1\right) \\
& -\sum_{r=n+1}^{2 m+1}\left(\sum_{\ell=1}^{m_{1}} \sum_{\gamma=m_{1}+1}^{n}\left(\zeta_{\ell \gamma}^{r}\right)^{2}+\sum_{t=2}^{m_{1}} \zeta_{11}^{r} \zeta_{t t}^{2}-\sum_{\ell=2}^{m_{1}} \sum_{\gamma=m_{1}+1}^{n} \zeta_{\ell \ell}^{r} \zeta_{\gamma \gamma}^{r}\right) \\
& -\frac{1}{2} \sum_{r=n+1}^{2 m+1}\left(2 \zeta_{11}^{r}-\left(\zeta_{m_{1}+1 m_{1}+1}^{r}+\cdots+\zeta_{n n}^{r}\right)\right)^{2} .
\end{aligned}
$$

As for the warped product submanifold $\Omega^{n}$ such that the base is minimal in $\Omega^{n}$, we compute the following simplification:

$$
\sum_{r=n+1}^{2 m+1} \sum_{\ell=2}^{m_{1}} \sum_{\gamma=m_{1}+1}^{n} \zeta_{\ell \ell}^{r} \zeta_{\gamma \gamma}^{r}=-\sum_{r=n+1}^{2 m+1} \sum_{\gamma=m_{1}+1}^{n} \zeta_{11}^{r} \zeta_{\gamma \gamma}^{r} .
$$

Similarly, we have

$$
\sum_{r=n+1}^{2 m+1} \sum_{t=2}^{m_{1}} \zeta_{11}^{r} \zeta_{t t}^{r}=-\sum_{r=n+1}^{2 m+1}\left(\zeta_{11}^{r}\right)^{2}
$$

At the same time, utilizing the minimality of the base manifold $\mathbb{N}_{1}$, we deduce that

$$
\begin{aligned}
& \frac{1}{2} \sum_{r=n+1}^{2 m+1}\left(2 \zeta_{11}^{r}-\left(\zeta_{m_{1}+1 m_{1}+1}^{r}+\cdots+\zeta_{n n}^{r}\right)\right)^{2}+\sum_{r=n+1}^{2 m+1} \sum_{\gamma=m_{1}+1}^{n} \zeta_{11}^{r} \zeta_{\gamma \gamma}^{r} \\
& \quad=2 \sum_{r=n+1}^{2 m+1}\left(\zeta_{11}^{r}\right)^{2}+\frac{1}{2} n^{2}\|\mathbb{H}\|^{2} .
\end{aligned}
$$


Utilizing (2.35), (2.36) and (2.37), (2.34), will lead to

$$
\mathscr{R} i c(\mathbb{W}) \leq \frac{\epsilon}{4}\left(m_{1} m_{2}+n-1\right)-\frac{m_{2} \Delta f}{f}+\sum_{r=n+1}^{2 m+1}\left\{\sum_{\gamma=m_{1}+1}^{n} \zeta_{11}^{r} \zeta_{\gamma \gamma}^{r}-\left(\zeta_{11}\right)^{2}\right\}
$$

The above inequality is equivalent to the following:

$$
\mathscr{R} i c(\mathbb{W}) \leq \frac{\epsilon}{4}\left(m_{1} m_{2}+n-1\right)-\frac{m_{2} \Delta f}{f}+\sum_{r=n+1}^{2 m+1} \sum_{\gamma=2}^{n} \zeta_{11}^{r} \zeta_{\gamma \gamma}^{r}
$$

Using (2.20) gives inequality (2.21).

Case II. Assume that $e_{u}$ is tangent $\mathbb{N}_{2}$. Fix a unit tangent vector field from $e_{m_{1}+1}, \ldots, e_{n}$ in which $\mathbb{W}=e_{u}=e_{n}$. Utilizing (2.14)-(2.29) and following a similar technique as in Case I implies

$$
\begin{aligned}
\frac{1}{2} n^{2}\|\mathbb{H}\|^{2} \geq & \mathscr{R} i c(\mathbb{W})+\frac{m_{2} \Delta f}{f}-2 \tilde{\tau}\left(T_{x} \Omega^{n}\right)+\tilde{\tau}\left(T_{x} \mathbb{N}_{1}^{m_{1}}\right)+\tilde{\tau}\left(T_{x} \mathbb{N}_{2}^{m_{2}}\right) \\
& +\sum_{1 \leq \ell<\gamma \leq n-1} \tilde{\mathbb{K}}_{\ell \gamma}+\frac{1}{2} \sum_{r=n+1}^{2 m+1}\left(2 \zeta_{n n}^{r}-\left(\zeta_{m_{1}+1 m_{1}+1}^{r}+\cdots+\zeta_{n n}^{r}\right)\right)^{2} \\
& +\sum_{r=n+1}^{2 m+1} \sum_{\gamma=1}^{n-1} \zeta_{n n}^{r} \zeta_{\gamma \gamma}^{r}+\sum_{r=n+1}^{m} \sum_{\ell=1}^{m_{1}} \sum_{\gamma=m_{1}+1}^{n}\left(\zeta_{\ell \gamma}^{r}\right)^{2}-\sum_{r=n+1}^{2 m+1} \sum_{\ell=1}^{m_{1}} \sum_{\gamma=m_{1}+1}^{n-1} \zeta_{\ell \ell}^{r} \zeta_{\gamma \gamma}^{r} .
\end{aligned}
$$

Using (2.30), we obtain

$$
\begin{aligned}
\mathscr{R} i c(\mathbb{W}) \leq & \frac{1}{2} n^{2}\|\mathbb{H}\|^{2}-\frac{m_{2} \Delta f}{f}+\frac{\epsilon}{4}\left(m_{1} m_{2}+n-1\right) \\
& -\frac{1}{2} \sum_{r=n+1}^{2 m+1}\left(2 \zeta_{n n}^{r}-\left(\zeta_{m_{1}+1 m_{1}+1}^{r}+\cdots+\zeta_{n n}^{r}\right)\right)^{2}-\sum_{r=n+1}^{2 m+1} \sum_{\gamma=1}^{n-1} \zeta_{n n}^{r} \zeta_{\gamma \gamma}^{r} \\
& -\sum_{r=n+1}^{2 m+1} \sum_{\ell=1}^{m_{1}} \sum_{\gamma=m_{1}+1}^{n}\left(\zeta_{\ell \gamma}^{r}\right)^{2}+\sum_{r=n+1}^{2 m+1} \sum_{\ell=1}^{m_{1}} \sum_{\gamma=m_{1}+1}^{n-1} \zeta_{\ell \ell}^{r} \zeta_{\gamma \gamma}^{r} .
\end{aligned}
$$

As the base of $\Omega^{n}$ is minimal,

$$
\sum_{r=n+1}^{2 m+1} \sum_{\ell=1}^{m_{1}} \sum_{\gamma=m_{1}+1}^{n-1} \zeta_{\ell \ell}^{r} \zeta_{\ell \gamma}^{r}=0
$$

By a similar technique from first case, using (2.41) in (2.40), we get

$$
\begin{aligned}
\mathscr{R} i c(\mathbb{W})+\frac{m_{2} \Delta f}{f} \leq & \frac{1}{2} n^{2}\|\mathbb{H}\|^{2}+\frac{\epsilon}{4}\left(m_{1} m_{2}+n-1\right) \\
& -\frac{1}{2} \sum_{r=n+1}^{2 m+1}\left(2 \zeta_{n n}^{r}-\left(\zeta_{m_{1}+1 q+1}^{r}+\cdots+\zeta_{n n}^{r}\right)\right)^{2} \\
& -\sum_{r=n+1}^{2 m+1} \sum_{\gamma=1}^{n-1} \zeta_{n n}^{r} \zeta_{\gamma \gamma}^{r}-\sum_{r=n+1}^{m} \sum_{\ell=1}^{m_{1}} \sum_{\gamma=m_{1}+1}^{n}\left(\zeta_{\ell \gamma}^{r}\right)^{2} .
\end{aligned}
$$


After some calculations, we obtain

$$
\begin{aligned}
\sum_{r=n+1}^{2 m+1} & \left\{\frac{1}{2}\left(\left(\zeta_{m_{1}+1 m_{1}+1}^{r}+\cdots+\zeta_{n n}^{r}\right)-2 \zeta_{n n}^{r}\right)^{2}+\sum_{\gamma=n+1}^{n-1} \zeta_{n n}^{r} \zeta_{\gamma \gamma}\right\} \\
= & \sum_{r=n+1}^{2 m+1} \frac{1}{2}\left(\zeta_{m_{1}+1 m_{1}+1}^{r}+\cdots+\zeta_{n n}^{r}\right)^{2}+2 \sum_{r=n+1}^{2 m+1}\left(\zeta_{n n}^{r}\right)^{2} \\
& -\sum_{r=n+1}^{2 m+1} \sum_{\gamma=m_{1}+1}^{n} \zeta_{n n}^{r} \zeta_{\gamma \gamma}^{r}+\sum_{r=n+1}^{m} \sum_{\gamma=n+1}^{n-1} \zeta_{n n}^{r} \zeta_{\gamma \gamma}-\sum_{r=n+1}^{2 m+1} \sum_{\gamma=m_{1}+1}^{n} \zeta_{n n}^{r} \zeta_{\gamma \gamma}^{r}
\end{aligned}
$$

Performing more calculations on the last two terms gives

$$
\sum_{r=n+1}^{2 m+1} \sum_{\gamma=n+1}^{n-1} \zeta_{n n}^{r} \zeta_{\gamma \gamma}-\sum_{r=n+1}^{2 m+1} \sum_{\gamma=m_{1}+1}^{n} \zeta_{n n}^{r} \zeta_{\gamma \gamma}^{r}=-\sum_{r=n+1}^{2 m+1}\left(\zeta_{n n}^{r}\right)^{2}
$$

Thus (2.43) can be reduced, using the above relation, to

$$
\begin{aligned}
& \sum_{r=n+1}^{2 m+1}\left\{\frac{1}{2}\left(\left(\zeta_{m_{1}+1 m_{1}+1}^{r}+\cdots+\zeta_{n n}^{r}\right)-2 \zeta_{n n}^{r}\right)^{2}+\sum_{\gamma=m_{1}+1}^{n-1} \zeta_{n n}^{r} \zeta_{\gamma \gamma}\right\} \\
& \quad=\sum_{r=n+1}^{2 m+1}\left\{\frac{1}{2}\left(\zeta_{m_{1}+1 m_{1}+1}^{r}+\cdots+\zeta_{n n}^{r}\right)^{2}+\left(\zeta_{n n}^{r}\right)^{2}-\sum_{\gamma=m_{1}+1}^{n-1} \zeta_{n n}^{r} \zeta_{\gamma \gamma}\right\}
\end{aligned}
$$

Therefore, using (2.44) in inequality (2.42), we deduce that

$$
\begin{aligned}
\mathscr{R} i c(\mathbb{W}) \leq & \frac{1}{2} n^{2}\|\mathbb{H}\|^{2}+\frac{\epsilon}{4}\left(m_{1} m_{2}+n-1\right)-\frac{m_{2} \Delta f}{f}-\frac{1}{4} \sum_{r=n+1}^{2 m+1}\left(\zeta_{m_{1}+1 m_{1}+1}^{r}+\cdots+\zeta_{n n}^{r}\right)^{2} \\
& -\sum_{r=n+1}^{2 m+1}\left\{\left(\zeta_{n n}^{r}\right)^{2}-\sum_{\gamma=m_{1}+1}^{n-1} \zeta_{n n}^{r} \zeta_{\gamma \gamma}+\frac{1}{4}\left(\zeta_{m_{1}+1 m_{1}+1}^{r}+\cdots+\zeta_{n n}^{r}\right)^{2}\right\} .
\end{aligned}
$$

From the minimality of the base of the warped product submanifold $\Omega^{n}$, we get

$$
\mathscr{R} i c(X) \leq \frac{\epsilon}{4}\left(m_{1} m_{2}+n-1\right)-\frac{m_{2} \Delta f}{f}-\sum_{r=n+1}^{2 m+1}\left(\zeta_{n n}^{r}-\sum_{\gamma=m_{1}+1}^{n-1} \zeta_{n n}^{r} \zeta_{\gamma \gamma}\right)
$$

This gives the proof of inequality (2.21). We will use the technique adopted for case (1) to get inequality (2.21) when $\Omega^{n}$ is $\mathbb{N}_{2}$-minimal. Now equality (2.21) can be verified similarly as in $[3,4,29]$.

For completely minimal submanifolds, Lemma 2.1 will lead to the following result.

Lemma 2.2 Assume $\omega: \Omega^{n}=\mathbb{N}_{1} \times_{f} \mathbb{N}_{2} \longrightarrow \widetilde{\mathbb{M}}^{2 m+1}$ is a $C$-totally real minimal isometric embedding of a warped product $\Omega^{n}$ to the cosymplectic space form $\tilde{\mathbb{M}}^{2 m+1}$. Then, for any 
unit vector $\mathbb{W} \in T_{x} \Omega^{n}$, the following Ricci inequality is satisfied:

$$
\mathscr{R} i c(\mathbb{W})+m_{2} \Delta \ln f \leq m_{2}\|\nabla \ln f\|^{2}+\frac{\epsilon}{4}\left\{m_{1} m_{2}+n-1\right\},
$$

where $m_{1}=\operatorname{dim} \mathbb{N}_{1}$ and $m_{2}=\operatorname{dim} \mathbb{N}_{2}$.

\section{Proof of the main results}

\subsection{Proof of Theorem 1.1}

Consider the following equation with $\omega=\ln f$ :

$$
|\operatorname{Hess}(\omega)+t \omega I|^{2}=|\operatorname{Hess}(\omega)|^{2}+t^{2}(\omega)^{2}|I|^{2}+2 t \omega g(\operatorname{Hess}(\omega), I) .
$$

But we know that $|I|^{2}=\operatorname{trace}\left(I I^{*}\right)=m_{1}$ and $g\left(\operatorname{Hess}(\omega), I^{*}\right)=\operatorname{tr}\left(\operatorname{Hess}(\omega) I^{*}\right)=\operatorname{trHess}(\omega)$. Then the preceding equation takes the form

$$
|\operatorname{Hess}(\omega)+t \omega I|^{2}=|\operatorname{Hess}(\omega)|^{2}+m_{1} t^{2}(\omega)^{2}-2 t \omega \Delta \omega .
$$

If $\lambda_{1}$ is an eigenvalue of the eigenfunction $\omega$, then $\Delta \omega=\lambda_{1} \omega$. Thus we get

$$
|H e s s(\omega)+t \omega I|^{2}=|\operatorname{Hess}(\omega)|^{2}+\left(m_{1} t^{2}-2 t \lambda_{1}\right)(\omega)^{2} .
$$

On the other hand, we obtain

$$
\Delta \frac{\omega^{2}}{2}=\omega \Delta \omega-|\nabla \omega|^{2} .
$$

Again using $\Delta \omega=\lambda_{1} \omega$, with integration, we arrive at

$$
\frac{\Delta \omega^{2}}{2}=\omega \lambda_{1} \omega-|\nabla \omega|^{2}
$$

which implies that

$$
\int_{\Omega^{n}} \omega^{2} d V=\frac{1}{\lambda_{1}} \int_{\Omega^{n}}|\nabla \omega|^{2} d V
$$

It follows from (3.2) and (3.3) that

$$
\int_{\Omega^{n}}|\operatorname{Hess}(\omega)+t \omega I|^{2} d V=\int_{\Omega^{n}}|\operatorname{Hess}(\omega)|^{2} d V+\int_{\Omega^{n}}\left(\frac{m_{1} t^{2}}{\lambda_{1}}-2 t\right)|\nabla \omega|^{2} .
$$

In particular, taking $t=\frac{\lambda_{1}}{m_{1}}$ in (3.4) and integrating, we get

$$
\int_{\Omega^{n}}\left|\operatorname{Hess}(\omega)+\frac{\lambda_{1}}{m_{1}} \omega I\right|^{2} d V=\int_{\Omega^{n}}|\operatorname{Hess}(\omega)|^{2} d V-\frac{\lambda_{1}}{m_{1}} \int_{\Omega^{n}}|\nabla \omega|^{2} d V .
$$

Again integrating (2.21) and involving the Green lemma, we have

$$
\begin{aligned}
\int_{\Omega^{n}} \mathscr{R} i c_{M}(\mathbb{W}) d V \leq & \frac{n^{2}}{4} \int_{\Omega^{n}}|\mathbb{H}|^{2} d V+m_{2} \int_{\Omega^{n}}|\nabla \omega|^{2} d V \\
& +\int_{\Omega^{n}} \frac{\epsilon}{4}\left(m_{1} m_{2}+n-1\right) d V .
\end{aligned}
$$


From (3.5) and (3.6), we derive

$$
\begin{aligned}
\frac{1}{q} \int_{\Omega^{n}} \mathscr{R i c}(\mathbb{W}) d V \leq & \frac{n^{2}}{4 m_{2}} \int_{\Omega^{n}}|\mathbb{H}|^{2} d V-\frac{m_{1}}{\lambda_{1}} \int_{\Omega^{n}}\left|\operatorname{Hess}(\omega)+\frac{\lambda_{1}}{n} \omega I\right|^{2} d V \\
& +\frac{m_{1}}{\lambda_{1}} \int_{\Omega^{n}}|\operatorname{Hess}(\omega)|^{2} d V+\int_{\Omega^{n}} \frac{\epsilon}{4}\left(m_{1}+1+\frac{m_{1}-1}{m_{2}}\right) d V .
\end{aligned}
$$

Under the assumption that the Ricci curvature is greater than or equal to zero, i.e., $\mathscr{R}$ ic $(\mathbb{W}) \geq 0$, the latter equation implies

$$
\begin{aligned}
\int_{\Omega^{n}}\left|\operatorname{Hess}(\omega)+\frac{\lambda_{1}}{m_{1}} \omega I\right|^{2} d V \leq & \frac{n^{2} \lambda_{1}}{4 m_{1} m_{2}} \int_{\Omega^{n}}|\mathbb{H}|^{2} d V+\int_{\Omega^{n}}|\operatorname{Hess}(\omega)|^{2} d V \\
& +\frac{\lambda_{1}}{m_{1}} \int_{\Omega^{n}} \frac{\epsilon}{4}\left(m_{1}+1+\frac{m_{1}-1}{m_{2}}\right) d V
\end{aligned}
$$

which is equivalent to the following:

$$
\begin{aligned}
\int_{\Omega^{n}}\left|\operatorname{Hess}(\omega)+\frac{\lambda_{1}}{m_{1}} \omega I\right|^{2} d V \leq & \int_{\Omega^{n}}|\operatorname{Hess}(\omega)|^{2} d V \\
& +\frac{\lambda_{1}}{4 m_{1}} \int_{\Omega^{n}}\left\{\frac{n^{2}}{m_{2}}|\mathbb{H}|^{2}+\epsilon\left(m_{1}+1+\frac{m_{1}-1}{m_{2}}\right)\right\} d V .
\end{aligned}
$$

If the following equality holds by assumption

$$
\int_{\Omega^{n}}|\operatorname{Hess}(\omega)|^{2} d V=\frac{\lambda_{1}}{4 m_{1} m_{2}} \int_{\Omega^{n}}\left\{\epsilon\left(1-n-m_{1} m_{2}\right)-n^{2}|\mathbb{H}|^{2}\right\} d V,
$$

then equations (3.7) and (3.8) imply that

$$
\int_{\Omega^{n}}\left|\operatorname{Hess}(\omega)+\frac{\lambda_{1}}{m_{1}} \omega I\right|^{2} d V \leq 0
$$

But it is clear that

$$
\int_{\Omega^{n}}\left|\operatorname{Hess}(\omega)+\frac{\lambda_{1}}{m_{1}} \omega I\right|^{2} d V \geq 0
$$

Combining Eqs. (3.9) and (3.10), we get

$$
\left|\operatorname{Hess}(\omega)+\frac{\lambda_{1}}{n} \omega I\right|^{2}=0 \quad \Longrightarrow \quad \operatorname{Hess}(\omega)=-\frac{\lambda_{1}}{m_{1}} \omega I .
$$

Since the WF $\omega=\ln f$ of the nontrivial WPS $\Omega^{n}$ is nonconstant, Eq. (3.11) reduces to Obata's differential equation where $c=\sqrt{\frac{\lambda_{1}}{m_{1}}}>0$ with $\lambda_{1}>0$. Hence, $\mathbb{N}_{1}$ is isometric to $\mathbb{S}^{p}\left(\sqrt{\frac{\lambda_{1}}{m_{1}}}\right)$. This completes the proof of the first part. On the other hand, if we have $\lambda_{1}=m_{1}$, then from (3.11) we get

$$
\operatorname{Hess}(\omega)\left(\mathbb{W}_{1}, \mathbb{W}_{2}\right)=-\omega g\left(\mathbb{W}_{1}, \mathbb{W}_{2}\right)
$$

for any $\mathbb{W}_{1}, \mathbb{W}_{2} \in \Gamma\left(\mathbb{N}_{1}\right)$. The proof of this theorem is now complete. 


\subsection{Proof of Theorem 1.2}

If $\omega$ is a positive differentiable function on a Riemannian manifold $\mathbb{N}_{1}$, then Bochner formula is given as

$$
\frac{1}{2} \Delta|\nabla \omega|^{2}=|\operatorname{Hess}(\omega)|^{2}+\mathscr{R} i c(\nabla \omega, \nabla \omega)+g(\nabla \omega, \nabla \Delta \omega) .
$$

Integrating along the volume element and using Stokes' theorem, we get

$$
\int_{\Omega^{n}}\left\{|\operatorname{Hess}(\omega)|^{2}+\mathscr{R} i c(\nabla \omega, \nabla \omega)+g(\nabla \omega, \nabla \Delta \omega)\right\} d V=0 .
$$

Assuming that $\lambda_{1}$ is an eigenvalue of the eigenfunction $\omega$ with $\Delta \omega=\lambda_{1} \omega$, we can conclude that

$$
\int_{\Omega^{n}}|\operatorname{Hess}(\omega)|^{2}=-\int_{\Omega^{n}} \mathscr{R} i c(\nabla \omega, \nabla \omega) d V-\lambda_{1} \int_{\Omega^{n}}|\nabla \omega|^{2} d V
$$

Inserting the above equation into (3.5), we achieve

$$
\int_{\Omega^{n}}\left|\operatorname{Hess}(\omega)+\frac{\lambda_{1}}{m_{1}} \omega I\right|^{2} d V=-\int_{\Omega^{n}} \mathscr{R} i c(\nabla \omega, \nabla \omega) d V-\lambda_{1}\left(\frac{m_{1}+1}{m_{1}}\right) \int_{\Omega^{n}}|\nabla \omega|^{2} d V .
$$

Utilizing (3.6) in the above equality, we arrive at

$$
\begin{gathered}
\frac{1}{q} \int_{\Omega^{n}} \operatorname{Ric}(\mathbb{W}) d V+\frac{m_{1}}{\lambda_{1}\left(m_{1}+1\right)} \int_{\Omega^{n}}\left|\operatorname{Hess}(\omega)+\frac{\lambda_{1}}{m_{1}} \omega I\right|^{2} d V \\
\quad+\frac{m_{1}}{\lambda_{1}\left(m_{1}+1\right)} \int_{\Omega^{n}} \mathscr{R} i c(\nabla \omega, \nabla \omega) d V \\
\leq \frac{n^{2}}{4 m_{2}} \int_{\Omega^{n}}|\mathbb{H}|^{2} d V+\frac{\left(m_{1} m_{2}+n-1\right)}{m_{2}} \int_{\Omega^{n}} \frac{\epsilon}{4} d V .
\end{gathered}
$$

It can be simplified as

$$
\begin{aligned}
\int_{\Omega^{n}}\left|\operatorname{Hess}(\omega)+\frac{\lambda_{1}}{m_{1}} \omega I\right|^{2} d V+\frac{\lambda_{1}\left(m_{1}+1\right)}{m_{1} m_{2}} \int_{\Omega^{n}} \mathscr{R} i c(\mathbb{W}) d V+\int_{\Omega^{n}} \mathscr{R} i c(\nabla \omega, \nabla \omega) d V \\
\leq \frac{\lambda_{1} n^{2}\left(m_{1}+1\right)}{4 m_{1} m_{2}} \int_{\Omega^{n}}|\mathbb{H}|^{2} d V \\
\quad+\frac{\lambda_{1}\left(m_{1} m_{2}+n-1\right)\left(m_{1}+1\right)}{m_{1} m_{2}} \int_{\Omega^{n}} \frac{\epsilon}{4} d V
\end{aligned}
$$

Following our assumption that the Ricci curvature is nonnegative, i.e., Ric $\geq 0$, we derive

$$
\int_{\Omega^{n}}\left|\operatorname{Hess}(\omega)+\frac{\lambda_{1}}{m_{1}} \omega I\right|^{2} d V \leq \frac{\lambda_{1}\left(m_{1}+1\right)}{4 m_{1} m_{2}} \int_{\Omega^{n}}\left\{n^{2}|\mathbb{H}|^{2}+\epsilon\left(m_{1} m_{2}+n-1\right)\right\} d V .
$$

By the hypothesis, the extrinsic condition (1.4) holds, thus

$$
\operatorname{Hess}(\omega)\left(\mathbb{W}_{1}, \mathbb{W}_{2}\right)=-\frac{\lambda_{1}}{m_{1}} \omega g\left(\mathbb{W}_{1}, \mathbb{W}_{2}\right),
$$


for any $\mathbb{W}_{1}, \mathbb{W}_{2} \in \Gamma\left(\mathbb{N}_{1}\right)$. This is again Obata's ODE [30] which implies that the base $\mathbb{N}_{1}$ is isometric to the Euclidean sphere $\mathbb{S}^{m_{1}}\left(\sqrt{\frac{\lambda_{1}}{m_{1}}}\right)$. The proof is completed.

Using the fact that the warped product submanifold $\Omega^{n}$ is minimal, we give the following corollary of Theorem 1.1.

Corollary 3.1 Let $\mathbb{M}^{2 m+1}(\epsilon)$ be a cosymplectic space form and $\Upsilon: \Omega^{n}=\mathbb{N}_{1} \times_{f} \mathbb{N}_{2} \longrightarrow$ $\mathbb{M}^{2 m+1}(\epsilon)$ be a C-totally real minimal isometric embedding of the warped product submanifold $\Omega^{n}$ into $\mathbb{M}^{2 m+1}(\epsilon)$ with a nonnegative Ricci curvature. Then, there is an isometry between the compact base $\mathbb{N}_{1}$ and the sphere $\mathbb{S}^{m_{1}}$ if the following is true:

$$
|\operatorname{Hess}(\omega)|^{2}=\frac{1}{2 m_{2}}\left\{\epsilon\left(1-n-\lambda_{1} m_{2}\right)\right\} .
$$

Proof Assuming $\Upsilon$ is minimal and $\lambda_{1}=m_{1}$, from (3.7), we get

$$
\begin{aligned}
\int_{\Omega^{n}}|\operatorname{Hess}(\omega)+\omega I|^{2} d V \leq & \int_{\Omega^{n}}|\operatorname{Hess}(\omega)|^{2} d V \\
& +\frac{1}{2} \int_{\Omega^{n}} \epsilon\left(\lambda_{1}+1+\frac{\lambda_{1}-1}{m_{2}}\right) d V .
\end{aligned}
$$

If the assumption (3.16) holds, we get the following from (3.17):

$$
\operatorname{Hess}(\omega)\left(\mathbb{W}_{1}, \mathbb{W}_{2}\right)=-\omega g\left(\mathbb{W}_{1}, \mathbb{W}_{2}\right)
$$

for a nonconstant function $\omega=\ln f$. Hence, using the arguments as in [30] completes the proof of the corollary.

\section{Some physical applications}

In this section, we investigate the Dirichlet energy that satisfies the following for a compact submanifold $\Omega$ and differentiable function $\theta: \Omega \longrightarrow \mathbb{R}:$

$$
E(\theta)=\frac{1}{2} \int_{\Omega}\|\nabla \theta\|^{2} d V,
$$

where $d V$ is a volume element. From this motivation, we give the following corollary by combining (2.21) and (4.1).

Corollary 4.1 Let $\Omega^{n}=\mathbb{N}_{1} \times_{f} \mathbb{N}_{2}$ be a compact $C$-totally real warped product submanifold embedded into a cosymplectic space form $\mathbb{M}^{2 m+1}(\epsilon)$. Then

(i) The following inequality holds:

$$
E(\ln f) \geq \frac{1}{2 m_{2}} \int_{\Omega^{n}}\left\{\mathscr{R} i c(\mathbb{W})-\frac{n^{2}}{4}\|\mathbb{H}\|^{2}-\frac{\epsilon}{4}\left(m_{1} m_{2}+n-1\right)\right\} .
$$

(ii) If $\Omega^{n}$ is minimal then we have

$$
E(\ln f) \geq \frac{1}{2 m_{2}} \int_{\Omega^{n}}\left\{\mathscr{R} i c(\mathbb{W})-\frac{\epsilon}{4}\left(m_{1} m_{2}+n-1\right)\right\},
$$

where $E(\ln f)$ is the Dirichlet energy of the warping function $\ln f$. 


\section{Conclusions}

In brief, it is well known that a cosmological model of the universe consisting of a perfect fluid whose molecules are galaxies is a Robertson-Walker spacetime. For example, if $\mathbb{S}^{3}$ indicates a three-dimensional manifold with constant curvature $\kappa=-1,0,1$ and $\mathbb{I}$ denotes an open interval in the real line $\mathbb{R}$, then a warped product of the form $\Omega(\kappa, f)=\mathbb{I} \times_{f} \mathbb{S}^{3}$ with its metric $d s^{2}=-d t^{2}+f^{2} d s_{\mathbb{S}}^{2}$ is a Robertson-Walker spacetime. Therefore, the concept of a warped product submanifold is useful because of its importance in mathematical physics $[5,6,12,16,18,19]$. In the present work, we have combined the ordinary differential equation with warped product submanifolds. Therefore, the paper presents excellent combinations of ordinary differential equation with Riemannian geometry.

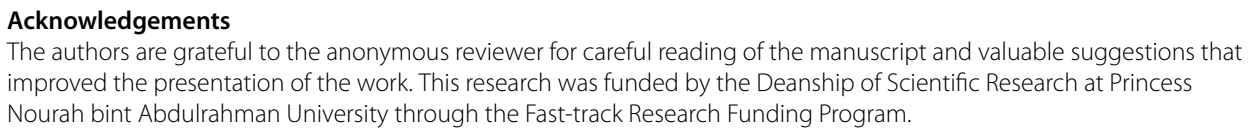

Availability of data and materials

There is no data available for this submission.

Competing interests

The authors declare that they have no competing interests.

Authors' contributions

All authors equally contribute for drafting of paper. All authors read and approved the final manuscript.

\section{Author details}

${ }^{1}$ Department of Mathematics, College of Science, King Khalid University, 61421 Abha, Saudi Arabia. ${ }^{2}$ Mathematical Science Department, Faculty of Science, Princess Nourah bint Abdulrahman University, 11546 Riyadh, Saudi Arabia. ${ }^{3}$ Institute of Mathematical Sciences, Faculty of Science, University of Malaya, Kuala Lumpur, Malaysia. ${ }^{4}$ Department of Applied Mathematics, Birla Institute of Technology, Mesra, Ranchi, India.

\section{Publisher's Note}

Springer Nature remains neutral with regard to jurisdictional claims in published maps and institutional affiliations.

Received: 3 July 2020 Accepted: 4 November 2020 Published online: 12 November 2020

\section{References}

1. Ali, A., Laurian-loan, P.: Geometry of warped product immersions of Kenmotsu space forms and its applications to slant immersions. J. Geom. Phys. 114, 276-290 (2017)

2. Ali, A., Laurian-loan, P.: Geometric classification of warped products isometrically immersed in Sasakian space forms. Math. Nachr. 292, 234-251 (2018)

3. Ali, A., Laurian-loan, P., Alkhaldi, A.H.: Ricci curvature on warped product submanifolds in spheres with geometric applications. J. Geom. Phys. 146, 103510 (2019)

4. Ali, A., Laurian-loan, P., Alkhaldi, A.H., Alqahtani, L.S.: Ricci curvature on warped product submanifolds of complex space forms and its applications. Int. J. Geom. Methods Mod. Phys. 16(9), 1950142 (2019)

5. Ali, A., Othman, W.A.M., Ozel, C.: Some inequalities for warped product pseudo-slant submanifolds of nearly Kenmotsu manifolds. J. Inequal. Appl. 2015, 291 (2015)

6. Ali, A., Othman, W.A.M., Ozel, C., Hajjari, T.: A geometric inequality for warped product pseudo-slant submanifolds of nearly Sasakian manifolds. C. R. Acad. Bulgare Sci. 70(2), 175-182 (2017)

7. Ali, A., Ozel, C.: Geometry of warped product pointwise semi-slant submanifolds of cosymplectic manifolds and its applications. Int. J. Geom. Methods Mod. Phys. 14(3), 1750042 (2017)

8. Ali, R., Mofarreh, F., Alluhaibi, N., Ali, A., Ahmad, I.: On differential equations characterizing Legendrian submanifolds of Sasakian space forms. Mathematics 8, 150 (2020)

9. Alluhaibi, N., Mofarreh, F., Ali, A., Mior Othman, W.A.: Geometric inequalities of warped product submanifolds and their applications. Mathematics 8(5), 759 (2020)

10. Alodan, H., Deshmukh, S., Turki, N.B., Vîlcu, G.E.: Hypersurfaces of a Sasakian manifold. Mathematics 8, $877(2020)$

11. Barros, A., Gomes, J.N., Ernani, J.R.: A note on rigidity of the almost Ricci soliton. Arch. Math. 100, 481-490 (2013)

12. Calin, O., Chang, D.C.: Geometric Mechanics on Riemannian Manifolds: Applications to Partial Differential Equations. Springer, Berlin (2006)

13. Chen, B.Y.: A general inequality for submanifolds in complex space forms and its applications. Arch. Math. 67, 519-528 (1996) 
14. Chen, B.Y:: Mean curvature and shape operator of isometric immersions in real-space-forms. Glasg. Math. J. 38, 87-97 (1996)

15. Chen, B.Y.: Relations between Ricci curvature and shape operator for submanifolds with arbitrary codimension. Glasg. Math. J. 41, 33-41 (1999)

16. Chen, B.Y.: On Ricci curvature of isotropic and Lagrangian submanifolds in complex space form. Arch. Math. (Basel) 74, 154-160 (2000)

17. Chen, B.Y.: On isometric minimal immersions from warped products into real space forms. Proc. Edinb. Math. Soc. 45, 579-587 (2002)

18. Chen, B.Y.: Pseudo-Riemannian Geometry, $\delta$-Invariants and Applications. World Scientific, Hackensack (2011)

19. Chen, B.Y.: Differential Geometry of Warped Product Manifolds and Submanifolds. World Scientific, Singapore (2017)

20. Deshmukh, S.: Conformal vector fields and eigenvectors of Laplace operator. Math. Phys. Anal. Geom. 15, 163-172 (2012)

21. Deshmukh, S.: Characterizing spheres and Euclidean spaces by conformal vector fields. Ann. Mat. Pura Appl. 196(6), 2135-2145 (2017)

22. Deshmukh, S.: Almost Ricci solitons isometric to spheres. Int. J. Geom. Methods Mod. Phys. 16(5), 1950073 (2019)

23. Deshmukh, S., Al-Eid, A.: Curvature bounds for the spectrum of a compact Riemannian manifold of constant scalar curvature. J. Geom. Anal. 15(4), 589-606 (2005)

24. Deshmukh, S., Al-Solamy, F.: A note on conformal vector fields on a Riemannian manifold. Colloq. Math. 136(1), 65-73 (2014)

25. Deshmukh, S., Al-Solamy, F.R.: Conformal gradient vector fields on a compact Riemannian manifold. Colloq. Math $112(1), 157-161(2008)$

26. Lee, J.W., Lee, C.W., Vilcu, G.E.: Classification of Casorati ideal Legendrian submanifolds in Sasakian space forms. J. Geom. Phys. 155, 103768 (2020)

27. Lichnerowicz, A.: Geometrie des Groupes de Transformations. Dunod, Paris (1958)

28. Mihai, I.: Ricci curvature of submanifolds in Sasakian space forms. J. Aust. Math. Soc. 72, 247-256 (2002)

29. Mustafa, A., Uddin, S., Al-Solamy, F.R.: Chen-Ricci inequality for warped products in Kenmotsu space forms and its applications. Rev. R. Acad. Cienc. Exactas Fís. Nat., Ser. A Mat. 113, 3585-3602 (2019)

30. Obata, M.: Certain conditions for a Riemannian manifold to be isometric with a sphere. J. Math. Soc. Jpn. 14, 333-340 (1962)

31. Sasahara, T.: A class of biminimal Legendrian submanifolds in Sasakian space forms. Math. Nachr. 287(1), 79-90 (2014)

32. Tashiro, Y.: Complete Riemannian manifolds and some vector fields. Trans. Am. Math. Soc. 117, 251-275 (1965)

33. Tripathi, M.M.: Improved Chen-Ricci inequality for curvature-like tensors and its applications. Differ. Geom. Appl. 29, 685-698 (2011)

34. Tripathi, M.M.: C-Totally real warped product submanifolds. An. Ştiinţ. Univ. 'Al.I. Cuza' laşi, Mat. 58, 417-436 (2012)

\section{Submit your manuscript to a SpringerOpen ${ }^{\circ}$ journal and benefit from:}

- Convenient online submission

- Rigorous peer review

- Open access: articles freely available online

- High visibility within the field

- Retaining the copyright to your article

Submit your next manuscript at $\gg$ springeropen.com 